\title{
COMPORTAMENTO ALIMENTAR E FATORES ASSOCIADOS EM SERVIDORES: CONTRIBUIÇÕES PARA A SAÚDE COLETIVA
}

\author{
FOOD BEHAVIOR AND FACTORS ASSOCIATED WITH SERVERS: CONTRIBUTIONS TO \\ COLLECTIVE HEALTH
}

Mariana Pimentel Gomes Souza ${ }^{\mathrm{a}^{*}}$

Orcid: http://orcid.org/0000-0002-7293-780X

Rafaella Sampaio ${ }^{\mathrm{b}^{*} \mathrm{e}^{* *}}$

Orcid: http://orcid.org/0000-0001-9994-1916
Soraia Pinheiro Machado Arruda $\mathrm{d}^{*}$

Orcid: http://orcid.org/0000-0002-3918-4738

Francisco José Maia Pinto ${ }^{\mathrm{e}^{*}}$

Orcid: https://orcid.org/0000-0002-4705-1513

Ana Carolina Montenegro Cavalcante $\mathrm{c}^{* *} \mathrm{e}^{* * *}$

Orcid: http://orcid.org/0000-0002-1086-0587

mariana_pimentelgomes@hotmail.comª,rafaellasampaio@yahoo.com.br ${ }^{\mathrm{b}}$, carolyna4481@hotmail.comc, soraia.arruda@uece.br ${ }^{\mathrm{d}}$, maiapinto@ yahoo.com.bre

Universidade Estadual do Ceará (UECE) ${ }^{*}$, Universidade de Fortaleza (UNIFOR) $)^{* *}$, Centro Universitário Unichristus ${ }^{* * *}$

Data de Submissão: 18/07/2019

Data de Aceite 18/12/2019

\section{RESUMO}

Introdução: $\mathrm{O}$ comportamento alimentar é definido como um conjunto de ações realizadas desde o momento da escolha do alimento a ser consumido. Comportamentos alimentares de risco podem desencadear o desenvolvimento de patologias e transtornos alimentares. Objetivo: Refletir e discutir sobre o comportamento alimentar e os fatores associados em servidores. Método: Trata-se de uma revisão narrativa realizada com base em periódicos nacionais e internacionais, abordando assuntos relacionados ao tema. As bases consultadas foram Bireme, LILACS, SciELO, Pubmed e portal de periódicos CAPES. Resultados: A pesquisa bibliográfica revelou que fatores relacionados ao estresse frequente, derivado da rotina e pressão em relação ao desempenho no trabalho, são indicativos de que alguns trabalhadores sejam um grupo susceptível a comportamentos alimentares de risco. A associação bidirecional entre qualidade de vida e desordens do comportamento alimentar vem sendo evidenciada e o estresse psicológico e a qualidade de vida referentes ao bem-estar mental estiveram relacionados com a piora dos sintomas de desordens alimentares. Além disso, revelou-se que sintomas de desordens alimentares contribuíram para intensificar o estresse psicológico e reduzir os níveis de qualidade de vida. Por meio do estudo reforça-se que os distúrbios no comportamento alimentar, provenientes de alterações psicológicas como ansiedade, estresse e atitudes alimentares, a fim de reduzir tensões, podem ocasionar o consumo excessivo de alimentos, resultando em obesidade. Conclusões: Assim, o ambiente de trabalho é o local ideal para o desenvolvimento de estudos investigativos, diagnósticos e de intervenção para doenças, pois o indivíduo passa a maior parte da sua vida nesse ambiente.

Palavras-chave: comportamento alimentar; estado nutricional; qualidade de vida; saúde coletiva.

\section{ABSTRACT}

Introduction: Eating behavior is defined as a set of actions performed from the moment of choosing the food to be consumed. Risk eating behaviors can trigger the development of eating disorders and disorders. Objective: To reflect and discuss the eating behavior and associated factors in servers. Method: This is a narrative review based on national and international journals, addressing issues related to the theme. The bases consulted were Bireme, LILACS, SciELO, Pubmed and CAPES journal portal. Results: The literature search revealed that factors related to frequent stress, derived from routine and pressure in relation to work performance, are indicative that some workers are a group susceptible to risky eating behaviors. The bidirectional association between quality of life and eating behavior disorders has been evidenced and psychological stress and quality of life related to mental well-being were related to the worsening symptoms of eating disorders. In addition, symptoms of eating disorders have been shown to contribute to intensifying psychological stress and reducing quality of life levels. Through the study it is reinforced that disturbances in eating behavior resulting from psychological changes such as anxiety, stress and eating attitudes in order to reduce tensions, can cause excessive food consumption resulting in obesity. Conclusions: Thus, the work environment is the ideal place for the development of investigative, diagnostic and intervention studies for diseases, since the individual spends most of his or her life in this environment.

Keywords: food behavior; nutritional status; quality of life; collective health. 


\section{Introdução}

O comportamento alimentar pode ser definido como um conjunto de ações realizadas desde o momento da escolha do alimento a ser consumido, de acordo com a disponibilidade e com o hábito alimentar. Além disso, as técnicas e os utensílios utilizados no momento do preparo dos alimentos, escolhidos segundo a cultura local, bem como os horários e as refeições realizadas também estão compreendidos no conceito de comportamento alimentar. Desse modo, pode-se dizer que o comportamento alimentar compreende todas as formas de convivência com o alimento. ${ }^{1}$ Abrange fatores relacionados à sensação de fome e saciedade, estados motivacionais e processos fisiológicos e metabólicos, que agem comandados pelos sistemas nervosos central e periférico. ${ }^{2}$

Comportamentos alimentares de risco podem desencadear o desenvolvimento de patologias e transtornos alimentares. Os transtornos alimentares são patologias de caráter psiquiátrico e debilitante, as quais consistem em distúrbios persistentes nos hábitos e no comportamento alimentar, o que acarreta problemas à saúde física e psicossocial do indivíduo. ${ }^{3}$ Atitudes que envolvam preocupação exacerbada com os alimentos, prática exagerada de exercícios físicos, uso de medicamentos no intuito de emagrecimento e restrição ou compulsão alimentar representam comportamentos de risco para o desenvolvimento de transtornos alimentares. ${ }^{4}$ A problemática e a prevalência dessas condições são globais e de grande relevância. ${ }^{5}$

Dentre os transtornos alimentares mais comuns estão anorexia nervosa, bulimia nervosa, transtorno de compulsão alimentar e transtorno alimentar restritivo. A anorexia nervosa está relacionada com a contínua restrição da ingesta alimentar em consequência do medo extremo de ganhar peso e alteração na percepção da própria imagem corporal. Associados a ela estão transtornos de caráter bipolar, depressivo e de ansiedade. Entre mulheres jovens, a prevalência de 12 meses é de aproximadamente 0,4\%. Já entre os indivíduos do sexo masculino, a prevalência é bem menor, com proporção feminino-masculino de aproximadamente 10:1. Já a bulimia nervosa se relaciona a episódios frequentes de compulsão alimentar, bem como atitudes compensatórias recorrentes inadequadas, visando evitar o ganho de peso e a distorção da imagem corporal. Associadas a esse transtorno são comuns sintomas depressivos. Entre jovens do sexo feminino, a prevalência de 12 meses é representada por 1 a $1,5 \%$. No gênero masculino, a prevalência também é bem menor e apresenta a mesma proporção feminino-masculino da anorexia nervosa. ${ }^{6}$

No transtorno alimentar restritivo, a característica diagnóstica mais expressiva é a restrição da ingestão alimentar, a qual é evidenciada pela não satisfação das necessidades energéticas por meio da ingestão oral de alimentos. Transtorno obsessivo-compulsivo e transtornos do neurodesenvolvimento, como déficit de atenção e transtorno autista estão geralmente associados ao comportamento restritivo. Já o transtorno de compulsão alimentar é caracterizado por episódios de ingestão alimentar exagerada em relação a ingestão alimentar que a maioria das pessoas consumiriam no mesmo espaço de tempo e em circunstâncias similares. Nesse contexto, o transtorno pode propiciar o ganho de peso e aumentar os riscos do desenvolvimento de obesidade. Além disso, é comum a associação a transtornos depressivos e de ansiedade, propiciando prejuízos à qualidade de vida do indivíduo. Em mulheres e homens norteamericanos com 18 anos ou mais, a prevalência de 12 meses é de 1,8 e $0,8 \%$, respectivamente. ${ }^{6}$

O consumo alimentar excessivo, influenciado por determinado comportamento alimentar e fatores associados, pode promover ou agravar o desenvolvimento da obesidade e, consequentemente, de doenças crônicas não transmissíveis associadas, com destaque para as cardiovasculares e o diabetes mellitus tipo $2 .{ }^{7}$ No estudo nacional, Sistema de Vigilância dos Fatores de Risco para Doenças Crônicas por Inquérito Telefônico (VIGITEL), que incluiu as 26 capitais brasileiras mais o Distrito Federal, mostrou frequência de sobrepeso de 52,5\% e frequência de obesidade de $17,9 \% .{ }^{8}$ Um estudo desenvolvido com funcionários de uma universidade pública do Rio de Janeiro relevou prevalência de 63,5\% para excesso de peso e $27,4 \%$ para obesidade. ${ }^{9}$

Os altos níveis de obesidade têm motivado o aumento das investigações a respeito dos fatores 
causais dessa condição, ou seja, por que que as pessoas comem e por que comem muito. ${ }^{10}$

As principais teorias psicológicas do comer em excesso incluem a ingestão restritiva, emocional e externa. O comportamento restritivo consiste na excessiva restrição da ingestão alimentar, mas que por vezes está associado a episódios de ingestão alimentar exacerbada. Enquanto que fatores externos como aparência, aroma e sabor do alimento desempenham forte influência na ingestão alimentar de comedores externos. ${ }^{11}$ Em relação à ingestão emocional, para Rotella et al., ${ }^{12}$ seu conceito envolve muito mais do que comportamento alimentar ou comer em excesso, engloba determinados sentimentos e emoções (depressão, solidão, etc.) que levam o indivíduo a sentir uma urgência em comer como forma de lidar com diferentes emoções.

É notório que um dos aspectos mais significativos e condicionantes do estilo de vida de um indivíduo é o trabalho e todos os aspectos que estão envolvidos nesse contexto. ${ }^{13}$ Fatores relacionados ao estresse frequente, derivados da rotina e pressão em relação ao desempenho no trabalho, são indicativos de que alguns trabalhadores e profissionais sejam um grupo susceptível a comportamentos alimentares de risco. Maiores prevalências para bulimia nervosa e transtorno da compulsão alimentar foram observadas em trabalhadores compreendidos no grupo de alta exigência, onde a atividade é realizada sob alta demanda psicológica e baixo controle. ${ }^{14} \mathrm{O}$ estresse foi relacionado positivamente com a ingestão calórica e a comportamentos alimentares não saudáveis. ${ }^{15}$ Estudo realizado com trabalhadores americanos revelou associação significante entre obesidade e longa jornada de trabalho (mais que 40 horas por semana), exposição à ambiente hostil de trabalho e insegurança no trabalho. ${ }^{16}$

Considerando a grande magnitude da obesidade, e a importante contribuição de comportamentos alimentares de risco para tal condição, e as inúmeras consequências negativas que estas condições oferecem, além das várias evidências a respeito da interferência de fatores ocupacionais na saúde física e mental e no estado nutricional dos indivíduos, é de extrema importância e relevância discutir sobre o comportamento alimentar e os fatores associados em servidores. Pois, desse modo, no âmbito da saúde coletiva espera-se contribuir no sentido de evidenciar aspectos relacionados ao comportamento alimentar inadequado, e, assim, incitar o planejamento e execução de intervenções que visem minimizar ou atenuar esses fatores de risco.

Sendo assim, o objetivo deste trabalho foi refletir e discutir acerca do comportamento alimentar e os fatores associados em servidores.

\section{Desenvolvimento}

\section{COMPORTAMENTO ALIMENTAR}

$\mathrm{O}$ ato de se alimentar envolve muito mais do que o simples objetivo de ingerir quantidades calóricas que irão satisfazer as necessidades do organismo. A ingestão alimentar é influenciada por inúmeros fatores, os quais podem ser conscientes ou não e que podem determinar escolhas e comportamentos do dia a dia. Dessa forma, o comportamento alimentar pode ser determinado pelo conjunto de práticas alimentares individuais ou coletivas. ${ }^{17}$

Ocomerrepresentaumato social, oqual fazparte do comportamento humano, e está relacionado a simbolismos, crenças e imaginação. Dessa forma, visto a complexidade de fatores envolvidos no contexto do comportamento alimentar, alterálo requer compreensão e superação das várias maneiras de convivência com os alimentos desde o nascimento e desenvolvimento. ${ }^{1}$

Experiências individuais e pessoais vivenciadas ao longo da vida contribuem para a definição de valores, tradições e crenças, as quais, somadas às influências sociais e ambientais inerentes à realidade de cada indivíduo exercem forte controle no estilo de vida e nos hábitos e comportamentos alimentares, bem como na ocorrência ou não de modificações dos mesmos. ${ }^{18}$ 
FATORES ASSOCIADOS AO COMPORTAMENTO ALIMENTAR

\section{ESTADO NUTRICIONAL E CONSUMO ALIMENTAR}

O comportamento alimentar em toda sua complexidade de fatores envolvidos e fatores influenciadores exerce forte e importante efeito no estado nutricional do indivíduo, seja na direção do sobrepeso e obesidade ou na perspectiva de perda de peso. ${ }^{19}$ Nesse contexto, as três mais estudadas esferas do comportamento alimentar: restrição alimentar, ingestão emocional e ingestão externa estão sendo intensamente investigadas no sentido de explicar o estado nutricional e as mudanças de peso corporal em indivíduos adultos. ${ }^{20}$

Essas três vertentes do comportamento alimentar mostraram contribuir de forma positiva em aumentar o IMC. No entanto, somente a ingestão emocional mostrou contribuir de forma significativa $(\mathrm{P}<0.05) .{ }^{21}$ Além disso, revelou-se o efeito da alta ingestão emocional detectada pelo DEBQ na quantidade da ingesta alimentar e nos tipos de alimentos consumidos posteriormente a episódios de tristeza ou alegria. Após a vivência de momentos de tristeza, comedores emocionais tendem a comer mais comparado a momentos de alegria ou prazer. Adicionalmente, evidenciou-se que após episódios de tristeza ou stress, comedores emocionais tendem a buscar a ingestão de alimentos de sabor doce comparados a alimentos salgados. $^{22}$

No entanto, estudo realizado com estudantes universitárias revelou que emoções negativas ou mau humor não exerce nenhum efeito na ingesta alimentar em comedores emocionais e não emocionais. Além disso, evidenciou-se que comedores emocionais quando encontram-se em momento de bom humor tendem a aumentar a ingesta alimentar quando comparado a momentos de humor neutro. Enquanto que comedores não emocionais mantêm aproximadamente a mesma ingesta alimentar em ambas as situações. ${ }^{23}$

Indivíduos obesos comparados a indivíduos ex-obesos que obtiveram sucesso na perda de peso apresentaram maiores percentagens de compulsão alimentar. ${ }^{24}$ Nesse sentido, verificouse que a regressão na compulsão alimentar pode levar à redução do IMC, sendo então o tratamento da compulsão alimentar um fator significativo para o sucesso na perda de peso e assim para vencer a obesidade. ${ }^{25} \mathrm{Em}$ relação à frequência do comportamento de restrição, o grupo de obesos e o grupo com perda de peso bem sucedida não apresentaram diferença. ${ }^{24}$ Isso ocorre possivelmente pelo fato da prática de comportamentos restritivos ser muito comum em indivíduos que preocupam-se com o peso e têm o objetivo de emagrecer e em obesos após episódios de compulsão alimentar. ${ }^{6}$

Estudos que investigaram a associação entre comportamentos alimentares e possíveis mudanças no IMC não encontraram nenhuma evidência clara do comportamento restritivo ser um fator de risco para o ganho de peso. No entanto, tal comportamento também não se mostrou claramente associado com a perda de peso. $^{21,26}$

Estudo realizado com mulheres universitárias revelou que indivíduos com alto nível de ingestão externa, identificada por meio da aplicação do DEBQ, consumiram mais alimentos não saudáveis de sabor doce durante o teste de consumo realizado em laboratório. ${ }^{27}$ Nesse sentido, estudo que investigou as razões que levam indivíduos sobrepesados e obesos a consumirem alimentos não saudáveis, como lanches ricos em açúcar e gordura revelou que a causa mais prevalente foi o cheiro e aparência tentadores, revelando assim a forte influência das características dos alimentos na ingestão externa. ${ }^{2}$

\section{QUALIDADE DE VIDA}

Diversos fatores e aspectos da vida devem ser levados em conta quando fala-se em qualidade de vida. Assim, para que o indivíduo consiga ter uma adequada qualidade de vida é interessante que ele se sinta confortável, satisfeito tanto na área pessoal e afetiva como na vida profissional e social. ${ }^{1}$ Em outras palavras, a qualidade de vida é um complexo indicador que tenta avaliar a capacidade de determinado indivíduo em estruturar e viver uma vida com condições adequadas. ${ }^{29}$

A qualidade de vida no contexto do trabalho deve englobar e pautar-se em aspectos como bem-estar no ambiente de trabalho, possibilidade de reconhecimento e crescimento profissional 
e respeito às características individuais. Nesse sentido, defende-se que a produtividade e o bem-estar devem estar associados no sentido de promover a chamada produtividade positiva e assim prover a satisfação no contexto do trabalho. ${ }^{30}$

O trabalho ocupa lugar de destaque na vida do homem, o qual pode ter sua percepção de qualidade de vida modificada a partir de impactos positivos ou negativos advindos do ambiente de trabalho. Desse modo, para que o contexto de trabalho possa proporcionar uma boa qualidade de vida ao indivíduo, este deve prover saúde e equilíbrio físico e emocional. ${ }^{31}$

Estudo com trabalhadores dos setores administrativos de empresas revelou que aspectos relacionados à qualidade do sono, tabagismo, quantidade de horas sentadas, relacionamento com a família e a prática de atividade física para fins estéticos interferem na qualidade de vida e saúde. ${ }^{29}$

As inúmeras e diferentes demandas advindas do trabalho podem, a médio e longo prazo, ocasionar alterações negativas sobre a qualidade de vida tanto social como psicológica do indivíduo, podendo resultar em estresse ocupacional..$^{32}$ Trabalhadores de uma indústria que apresentaram altos níveis de vulnerabilidade ao estresse relataram perceber alterações nos hábitos alimentares quando submetidos a situações de estresse. Adicionalmente, a prevalência da percepção de alterações alimentares em condições estressantes foi consideravelmente maior entre as mulheres, as quais relataram sentir mais fome e ter preferência por alimentos doces nessas situações. ${ }^{33}$

Esses achados corroboram com outro estudo, o qual evidenciou associação positiva entre estresse e consumo de doces, fast foods, alimentos enlatados e refrigerantes entre indivíduos do sexo feminino. Já em relação ao grupo de indivíduos do sexo masculino, o estresse associou-se positivamente somente com a ingestão de fast foods e alimentos enlatados. Enquanto que o consumo de saladas e vegetais crus esteve negativamente associado ao estresse para ambos os sexos. ${ }^{34}$

O estresse psicológico e a qualidade de vida referentes ao bem-estar mental estiveram relacionados com a piora dos sintomas de desordens alimentares. Além disso, revelou-se que sintomas de desordens alimentares contribuíram em intensificar o estresse psicológico e reduzir os níveis de qualidade de vida. ${ }^{35}$

A associação bidirecional entre qualidade de vida e desordens do comportamento alimentar vem sendo evidenciada. Revelou-se que os sintomas derivados de desordens alimentares e alto IMC preveem uma piora da qualidade de vida, enquanto que baixos níveis de qualidade de vida fisiológica (referentes à vitalidade, dores, limitações referentes a práticas de atividade física) a médio e longo prazo também preveem aumento da severidade e agravamento de desordens alimentares. ${ }^{35}$ Assim, considerando o impacto do baixo nível de qualidade de vida fisiológica nas desordens do comportamento alimentar, indivíduos obesos, os quais vivem em uma sociedade que o condiciona a preocuparse de forma exacerbada com a aparência física, podem apresentar episódios de insatisfação com a imagem corporal e assim episódios de padrões alimentares inadequados, como comportamentos predominantemente de compulsão alimentar pontuados por comportamentos de restrição. ${ }^{36}$

\section{FATORES OCUPACIONAIS}

A Saúde do Trabalhador é parte integrante da Saúde Pública e leva em consideração as interrelações entre trabalho, produção e saúde no âmbito social e ambiental, considerando que o trabalho é um significativo fator no que diz respeito ao processo saúde-doença. Defendese ainda que o trabalhador é sujeito ativo da sua história no contexto pessoal e laboral, e inclusive do desenvolvimento de melhorias nas circunstâncias de trabalho.

Nos dias atuais, a saúde do trabalhador pode ser afetada pelo ambiente competitivo, exigente, hostil e desgastante que muitas vezes está agregado ao contexto de trabalho do indivíduo. Dessa forma, em decorrência desses fatores, o processo de adoecimento do trabalhador pode iniciar-se devido a prejuízos na saúde física e psicológica. ${ }^{32}$

Fatores como o trabalho em tempo integral e longas horas de trabalho ( $>40 \mathrm{~h}$ por semana), mostraram-se associados ao aumento no risco de desenvolvimento de obesidade. Essa associação pode estar ligada ao fato de que indivíduos que trabalham por muitas horas possam vir a ter 
mais dificuldade em engajar-se em atividades físicas regulares. Adicionalmente, revelou-se que indivíduos que trabalham no horário da noite também são menos adeptos à prática de atividade física voltada ao fortalecimento muscular. ${ }^{37}$ Isso pode ser explicado ou influenciado pela associação entre trabalho noturno e a insuficiente qualidade do sono e consequente fadiga crônica. ${ }^{38}$

Turnos e horários de trabalho mostraramse influenciar também escolhas alimentares. Indivíduos do sexo feminino que trabalhavam em horários variantes mostraram maior proporção de energia derivada da ingestão de gordura saturada comparada a mulheres que trabalhavam durante o dia. Os indivíduos do sexo masculino que trabalhavam em turnos variantes, em relação aos que trabalhavam durante o dia, mostraramse menos propensos a consumir frutas e vegetais. Enquanto que o trabalho durante a noite mostrouse associado ao aumento do consumo de sacarose dos homens. ${ }^{39}$ Nesse sentido, a privação do sono vem sendo também relacionada a determinadas escolhas alimentares, como o aumento da ingestão de açúcar e lanches ricos em carboidrato. ${ }^{40}$

Estudo realizado com amostra composta por indivíduos do sexo feminino revelou que mulheres que relataram altos níveis de tensão no ambiente de trabalho apresentaram um significativo aumento no IMC, comparadas àquelas que relataram o contrário. Além disso, observou-se que as mulheres com mais alto IMC mostraram-se mais suscetíveis ao ganho de peso quando submetidas de forma contínua a altas exigências no trabalho. ${ }^{41}$

Estressores sociais, os quais referem-se à ocorrência de conflitos ou inimizades no ambiente de trabalho, e controle no trabalho, o que diz respeito à capacidade de autonomia ou tomada de decisões no contexto do trabalho, estiveram associados de forma positiva e significante com mudanças no IMC ao longo de dois anos. ${ }^{42}$ Intensas situações de estresse no trabalho mostraram-se associadas ao comer de forma exagerada, pôde-se notar isso em trabalhadores japoneses com níveis altos de IMC. ${ }^{43}$

$\mathrm{O}$ estresse apresenta-se como evento natural e próprio de ocorrer entre os seres humanos. No entanto, atualmente as inúmeras demandas advindas de diferentes esferas da vida têm transformado o estresse em um perigoso fator de risco para muitas patologias. ${ }^{44}$
Escassas são as pesquisas que abordam diretamente a influência de aspectos psicossociais de trabalho no comportamento alimentar ou no possível desenvolvimento de transtornos alimentares. No entanto, estudos demonstram associação entre estresse psicossocial no trabalho e transtornos mentais ${ }^{45} \mathrm{e}$ associação entre transtornos mentais e desordens alimentares. ${ }^{46}$

Sentimento de tensão no contexto laboral e trabalho de alta exigência e demanda estiveram fortemente associados ao aumento de sintomas depressivos ao longo de dois anos entre homens e mulheres. ${ }^{45}$ Nessesentido, mulheres diagnosticados com transtorno de compulsão alimentar sofreram efeito negativo sobre os sintomas da patologia por influência de sintomas depressivos derivados de problemas interpessoais. ${ }^{47}$ Desse modo, revelouse também que desordens alimentares estão associadas a comorbidades psiquiátricas como ansiedade, alteração de humor, ideações suicidas e transtornos neuróticos. ${ }^{48}$

Nesse contexto, desenvolveram-se técnicas na tentativa de prevenir ou atenuar doenças ocupacionais. Assim, a ginástica laboral surgiu e estabeleceu-se no contexto laboral no intuito de humanizá-lo, de romper com a monotonia e proporcionar um espaço de descontração em que os trabalhadores possam trabalhar simultaneamente corpo, cérebro e mente. Trabalhadores submetidos a sessões de ginástica laboral expressam redução da fadiga muscular, melhora na coordenação motora e na postura corporal, redução da fadiga mental e estresse, melhora na concentração, motivação e autoestima. ${ }^{31}$

Comparação feita entre trabalhadores participantes e não participantes do programa de ginástica laboral de uma Universidade revelou maior prevalência de inatividade física e percepção negativa de estresse no grupo de não participantes. Além disso, o grupo de mulheres submetidas à prática de ginástica laboral apresentou melhor satisfação com os colegas de trabalho comparado ao grupo não participante. ${ }^{49}$

\section{INSTRUMENTOS PARA AVALIAR O COMPORTA- MENTO ALIMENTAR}

Visto que atitudes alimentares englobam uma série de fatores como sentimentos, pensamentos, crenças e diferentes formas individuais de lidar 
com os alimentos referentes ao autocontrole, recusa alimentar, raiva, culpa, desejos e vergonha é interessante que os instrumentos de análise do comportamento alimentar abordem de forma satisfatória uma gama de aspectos que estão direta ou indiretamente relacionados à comida e ao ato de comer. ${ }^{50}$

O "Eating Behavior Inventory Questionnaire" (EBI) é um tipo de instrumento usado para avaliar comportamento alimentar. O mesmo é um instrumento de autorrelato desenvolvido no intuito de avaliar comportamentos teoricamente envolvidos na perda de peso, como automonitoramento do peso e da ingestão alimentar, recusas frente à oferta de determinados alimentos, alimentar-se em resposta a emoções, comer sempre em um mesmo local e realizar a compra de alimentos a partir de uma lista. $\mathrm{O}$ instrumento é composto por 30 itens, os quais foram estabelecidos na forma de afirmações em primeira pessoa, por exemplo: "Eu como no meio da noite". Dos 30 itens, 17 foram elaborados como afirmativas positivas ou facilitadoras em relação à perda de peso, como a seguinte afirmação: "Eu deixo comida no meu prato". No entanto, os 13 itens restantes foram formulados como afirmativas negativas ou não facilitadoras em relação à perda de peso, por exemplo: "Eu como, mesmo quando não estou realmente com fome". Assim, cada item é avaliado em uma escala de 5 pontos de acordo com a frequência que os fatos descritos nos itens ocorrem, usando as palavras 1- nunca ou quase nunca, 2- algumas vezes, 3- metade das vezes, 4- várias vezes, 5- sempre ou quase sempre. Em relação aos itens negativos, esses foram marcados na direção inversa da escala $(1=5,2=4)$. Dessa forma, os itens foram marcados no sentido de indicar ações apropriadas na perspectiva de controle ou perda de peso quanto mais alta for a pontuação. ${ }^{51}$

O EBI foi utilizado como instrumento para identificar mudanças comportamentais em relação à alimentação em 170 indivíduos sobrepesados e obesos que participaram de um estudo de intervenção para a perda de peso durante um ano. A análise revelou que os indivíduos que conseguiram manter a perda de peso durante o período de um ano demonstraram comportamentos alimentares mais favoráveis. ${ }^{52}$
O "Eating Attitudes Test" é outro tipo de instrumento utilizado para avaliar comportamento alimentar. É um instrumento de autorrelato, o qual é aplicado no sentido de identificar e avaliar padrões alimentares inapropriados ou anormais e como forma de acompanhar o avanço de casos clínicos. A versão original do instrumento (EAT40) foi validada a partir de uma amostra composta por dois grupos, um de mulheres canadenses portadoras de anorexia nervosa e outro de mulheres canadenses sem história da doença. Essa versão é constituída por 40 itens de múltipla escolha e o ponto de corte estabelecido foi de 30 pontos. Ou seja, se a soma final dos valores referentes às questões resultar em 30 ou mais é indicativo de possível distúrbio no comportamento alimentar. ${ }^{53}$

Já o EAT- 26 é uma versão reduzida baseada na versão original, mas que manteve a correlação entre pacientes e grupos de sujeitos normais. $\mathrm{O}$ questionário é constituído por 26 itens, os quais podem ser respondidos a partir de 6 opções de respostas, as quais possuem diferentes pontuações: sempre $=3$ pontos, muitas vezes $=2$ pontos, às vezes $=1$ ponto, poucas vezes $=0$ ponto, quase nunca $=0$ ponto e nunca $=0$ ponto. Desse modo, a análise fatorial é feita a partir de 3 aspectos, os quais englobam os itens. Os 13 primeiros itens refletem o fator 1 (dieta), abordando recusas caracterizadas como patológicas a alimentos muito calóricos e preocupação exacerbada com o corpo. Já os seis itens subsequentes refletem o fator 2 (bulimia nervosa), pois abordam questões referentes a comportamentos e pensamentos bulímicos em relação à comida. Enquanto que os sete itens restantes refletem o fator 3 (controle oral), abordando questões de autocontrole frente à comida e de pressões sociais inerentes ao ambiente do indivíduo. Assim, se o escore encontrado totalizar mais que 21 o resultado é considerado positivo e consequentemente há a confirmação da ocorrência de padrões alimentares anormais e de risco para o desenvolvimento de transtornos do comportamento alimentar. ${ }^{54}$

O EAT (40 ou 26) foi identificado como o instrumento mais utilizado por estudos originados em diferentes países e também entre estudos nacionais que utilizaram questionários que investigaram comportamentos de risco para desenvolvimento de algum transtorno alimentar, principalmente em adolescentes. ${ }^{5}$ 
O "The Three Factor Eating Questionnaire" (TFEQ) representa outro modelo de instrumento de autorrelato, constituído por 51 itens e desenvolvido para mensurar e avaliar três diferentes dimensões do comportamento alimentar: "Restrição Cognitiva Alimentar", o qual é caracterizado pela exagerada restrição da ingesta alimentar na perspectiva de controle de peso, "Desinibição", caracterizado pelo comer exagerado frente à perda de controle em relação à ingestão combinada à extrema sensação de fome, "Sensibilidade à Fome", o qual representa o comer influenciado por aspectos emocionais. ${ }^{55}$

Derivado da versão original foi desenvolvida uma versão reduzida, a qual conta com 18 itens. Comparado à versão original esse é mais simples e prático de ser respondido devido ao reduzido número de itens, principalmente no contexto de estudos epidemiológicos, os quais muitas vezes requerem o preenchimento de muitos questionários. Os itens do TFEQ-R18 são respondidos em uma escala de 4 pontos (totalmente verdade, na maioria das vezes é verdade, na maioria das vezes é mentira, totalmente mentira). Quanto mais alta a pontuação final nas respectivas escalas mais expressivo é o comer restritivo, descontrolado ou emocional do indivíduo. ${ }^{56}$

Outra versão reduzida do instrumento constituída por 21 itens foi traduzida para o português e aplicada em uma amostra composta por funcionárias do Hospital das Clínicas de São Paulo. A versão do instrumento mostrou-se adequada para identificar e avaliar comportamentos alimentares de restrição cognitiva, descontrole alimentar e ingestão emocional. ${ }^{57}$

Em relação ao Dutch Eating Behaviour Questionnaire (DEBQ), esse foi desenvolvido no intuito de investigar e analisar três vertentes do comportamento alimentar: restrição alimentar, ingestão externa e ingestão emocional. $\mathrm{O}$ instrumento é de autoadministração e engloba 33 itens, os quais estão distribuídos nas dimensões de comportamento citadas acima e avaliados em uma escala de 5 pontos (nunca/raramente/às vezes/ frequentemente/muito frequentemente). ${ }^{58}$

O instrumento é destinado a indivíduos jovens e adultos portadores de determinados distúrbios no comportamento alimentar, como anorexia e bulimia nervosa, comer compulsivo e obesidade. No entanto, também pode ser utilizado no intuito de avaliar o estilo alimentar de indivíduos saudáveis ou grupos de indivíduos que frequentemente se submetem a dietas, como atletas e bailarinos. ${ }^{19}$

A versão original do questionário desenvolvida por Van Strien et al. ${ }^{58}$ foi adaptada e validada para a população inglesa por Wardle, ${ }^{59} \mathrm{o}$ qual demonstrou que o questionário é extremamente útil em identificar os estilos alimentares que caracterizam os pacientes diagnosticados com bulimia ou anorexia nervosa ou os pacientes sobrepesados. Assim, o instrumento mostrouse eficaz para avaliar pacientes com desordens alimentares e de peso. Posteriormente, o instrumento foi traduzido para o português e dois anos mais tarde foi validado e considerado adequado ao estudo do comportamento alimentar em população portuguesa por meio de uma amostra composta por 191 jovens e adultos de ambos os sexos. ${ }^{19}$

Estudo realizado com 1562 funcionários de um banco da Holanda utilizou o DEBQ no intuito de, através de sua aplicação, revelar diferentes comportamentos alimentares e posteriormente associá-los a fatores de estilo de vida e mudanças no IMC. ${ }^{21}$

O DEBQ foi também utilizado em estudo que comparou o comportamento alimentar, o estilo de vida, a compulsão alimentar e a história de peso entre indivíduos obesos e indivíduos que obtiveram sucesso na perda de peso, no intuito de identificar as diferenças desses aspectos nos distintos grupos. ${ }^{24}$

\section{Considerações finais}

Estudos com trabalhadores têm importância para a saúde coletiva uma vez que o processo de adoecimento repercute na qualidade de vida e em gastos para o sistema de saúde. Assim, o ambiente de trabalho é o local ideal para o desenvolvimento de estudos investigativos, diagnósticos e de intervenção para doenças, pois o indivíduo passa a maior parte da sua vida nesse ambiente. 


\section{Referências}

1. Philippi ST. Alimentação Saudável e o Redesenho da Pirâmide dos Alimentos. In: Pirâmide dos Alimentos: fundamentos básicos da nutrição. São Paulo: Manole, 2014. cap. 1.

2. Nunes MA, Appolinario JC, Galvão AL, Coutinho W. Psicobiologia do apetite: a regulação episódica do comportamento alimentar. In: Halford Jason CG. Transtornos Alimentares e Obesidade. Porto Alegre: Artmed, 2008. cap. 1, p. 17-30.

3. Mahan LK, Stump SE, Raymond JL. Nutrição nos Transtornos Alimentares. In: Schebendach JE. Krause: Alimentos, Nutrição e Dietoterapia. Rio de Janeiro: Elsevier, 2013. cap. 23, p. 489-506.

4. Schaal K, Tafflet M, Nassif H, Thibault V, Pichard C, Alcotte M, Guillete T, Helou NE, Berthelot G, Simon S, Toussaint JF. Psychological Balance in High Level Athletes: Gender-Based Differences and Sport-Specific Patterns. Psychopathology and High Level Sport. 2011;6(5):1-9.

5. Leal GVS, Philippi ST, Polacow VO, Cordás TA, Alvarenga MS. O que é comportamento de risco para transtornos alimentares em adolescentes. Jornal Brasileiro de Psiquiatria. 2013;62(1):62-75

6. American Psychiatric Association. Manual Diagnóstico e Estatístico de Transtornos Mentais. 5. ed. Porto Alegre, 2014. 938p.

7. World Health Organization. World Health Statistics. Geneva: WHO, 2013. 168p.

8. Brasil. Ministério da Saúde. Vigitel Brasil 2014. Vigilância de fatores de risco para doenças crônicas por inquérito telefônico. Brasília: Ministério da Saúde, 2015. 152p. Disponível em: <http://bvsms.saude.gov.br/bvs/ publicacoes/vigitel_brasil_2014.pdf>.

9. Costa MAP, Vasconcelos AGG, Fonseca MJM. Prevalência de obesidade, excesso de peso e obesidade abdominal e associação com prática de atividade física em uma universidade federal. Revista Brasileira de Epidemiologia. 2014;17(2).

10. Brogan A, Hevey D. Eating styles in the morbidly obese: Restraint eating, but not emotional and external eating, predicts dietary behavior. Psychology \& Health, Dublin. 2013;28(6):714-725.

11. Hou R, Mogg K, Bradley BP, Morris RM, Peveler R, Roefs A. External eating, impulsivity and attentional bias to food cues. Appetite. 2011;56:424-427.

12. Rotella F, Fioravanti G, Godini L, Mannucci E, Faravelli C, Ricca V. Temperament and emotional eating: A crucial relationship in eating disorders. Psychiatric Research. 2015;225:452-457.
13. Freitas JD, Timossi LS, Francisco AC. Relação entre a qualidade de vida percebida e a qualidade de vida avaliada pelo WHOQOL- 100 nos colaboradores da UTFPR Campus Ponta Grossa. Revista ADMpg Gestão Estratégica. 2011;4(1):1-6.

14. Prisco APK, Araújo TM, Almeida MMG, Santos KOB. Prevalência de transtornos alimentares em trabalhadores urbanos de município do Nordeste do Brasil. Ciência e Saúde Coletiva. 2013;18(4):1109-1118.

15. Barrington WE, Ceballos RM, Bishop SK, Mcgregor BA, Beresford SAA. Perceived Stress, Behavior, and Body Mass Index Among Adults Participating in a Worksite Obesity Prevention Program, Seattle, 2005-2007. Preventing Chronic Disease. 2012;9.

16. Luckhaupt SE, Cohen MA, Li J, Calvert GM. Prevalence of Obesity Among U.S. Workers and Associations with Occupational Factors. American Journal of Preventive Medicine. 2014;46(3):237-248.

17. Gonçalves JA, Moreira EAM, Trindade EBSM, Fiates GM. Transtornos alimentares na infância e na adolescência. Revista Paulista de Pediatria. 2013;31(1):96-103.

18. Motta DG, Motta CG, Campos RR. Teorias psicológicas da fundamentação do aconselhamento nutricional. In: Diez-Garcia RW, Cervato-Mancuso AM. Mudanças alimentares e educação nutricional. Rio de Janeiro: Guanabara Koogan, 2011. cap. 5, p. 53-64.

19. Viana V, Sinde S. Estilo alimentar: adaptação e validação do questionário holandês do comportamento alimentar. Psicologia: Teoria, Investigação e Prática. 2003;1:59-71.

20. Snoek HM, Engels RCME, Van Strien T, Otten R. Emotional, external and restrained eating behaviour and BMI trajectories in adolescence. Appetite. 2013;67:81-87.

21. Koenders PG, Van Strien T. Emotional eating, rather than lifestyle behavior, drives weight gain in a prospective study in 1562 employees. Journal of Occupational \& Environmental Medicine. 2011;53(11):1287-1293.

22. Van Strien T, Cebolla A, Etchemendy E, GutiérrezMaldonado J, Ferrer-García M, Botella C, Baños R. Emotional eating and food intake after sadness and joy. Appetite. 2013;66:20-25.

23. Bongers P, Jansen A, Havermans R, Roefs A, Nederkoorn C. Happy eating. The underestimated role of overeating in a positive mood. Appetite. 2013;67:74-80.

24. Leitão M, Pimenta F, Herédia T, Leal I. Comportamento alimentar, compulsão alimentar, história de peso e estilo de vida: diferenças entre pessoas com obesidade e com uma perda de peso bem-sucedida. Alimentos e Nutrição $=$ Brazilian Journal of Food and Nutrition, Araraquara. 2013;24(4):393-401. 
25. Grilo CM, Masheb RM, Wilson GT, Gueorguieva R, White MA. Cognitive-behavioral therapy, behavioral weight loss, and sequential treatment for obese patients with binge-eating disorder: A randomized controlled trial. Journal of Consulting and Clinical Psychology. 2011;79(5):675-685.

26. Van Strien T, Herman CP, Verheijden MW. Eating style, overeating and weight gain. A prospective 2-year followup study in a representative Dutch sample. Appetite. 2012;59(3):782-789.

27. Kakoschke N, Kemps E, Tiggemann M. External eating mediates the relationship between impulsivity and unhealthy food intake. Physiology \& Behavior. 2015;147:117-121.

28. Cleobury L, Tapper K. Reasons for eating 'unhealthy' snacks in overweight and obese males and females. Journal of Human Nutrition and Dietetics. 2014;27(4):333-341.

29. Grande AJ, Silva V, Manzzato L, Rocha TBX, Martins GC, Vilela Junior GB. Determinantes da qualidade de vida no trabalho: ensaio clínico controlado e randomizado por clusters. Revista Brasileira de Medicina do Esporte. 2013;19(5):371-375.

30. Ferreira M. Qualidade de Vida no Trabalho. In: Cattani AD, Holzmani L. Dicionário de Trabalho e Tecnologia. 2. ed. (revisada e ampliada). Porto Alegre, RS: Zouk, 2011.

31. Mendes RA, Leite N. Ginástica laboral: histórico, definição, classificações e objetivos. In: Ginástica laboral: princípios e aplicações práticas. São Paulo: Manole, 2012. cap. 1.

32. Fonseca ISS, Araújo TM. Prevalence of common mental disorders among industry workers in Bahia, Brasil. Revista Brasileira de Saúde Ocupacional. 2014;39(129):35-49.

33. Costa FD, Teo CRPA, Almeida JS. Vulnerabilidade ao estresse e alimentação: um estudo no contexto do trabalho. Scientia Medica. 2015;25(2).

34. Ansari WE, Adetunji H, Oskrochi R. Food and mental health: relationship between food and perceived stress and depressive symptoms among university students in the united kingdom. Central European Journal of Public Health, Prague. 2014;22(2):90-97.

35. Mitchison D, Morin A, Mond J, Slewa-Younan S, Hay P. The Bidirectional Relationship between Quality of Life and Eating Disorder Symptoms: A 9-Year CommunityBased Study of Australian Women. PLOS One. 2015;10(3).

36. Mond J, Mitchison D, Latner J, Hay P, Owen C, Rodgers B. Quality of life impairment associated with body dissatisfaction in a general population sample of women. BMC Public Health. 2013;13(920).

37. Chin DL, Nam S, Lee SJ. Occupational factors associated with obesity and leisure-time physical activity among nurses: A cross sectional study. International Journal of Nursing Studies. 2016;57:60-69.
38. Han K, Thinkoff AM, Geiger Brown J. Factors associated with work-related fatigue and recovery in hospital nurses working 12-hour shifts. Workplace Health \& Safety. 2014;62(10):409-414.

39. Hemio K, Puttonen S, Viitasalo K, Harma M, Peltonen $\mathrm{M}$, Lindström J. Food and nutrient intake among workers with different shift systems. Occupational and Environment Medicine. 2015;72(7):513- 520.

40. Heaht G, Roach GD, Dorrian J, Ferguson SA, Darwent $\mathrm{D}$, Sargent C. The effect of sleep restriction on snacking behaviour during a week of simulated shiftwork. Accident Analysis and Prevention. 2012;45:62-67.

41. Fujishiro K, Lawson CC, Hibert EL, Chavarro JE, RichEdwards JW. Job strain and changes in the body mass index among working women: a prospective study. International Journal of Obesity. 2015;39(9):1395-1400.

42. Berset M, Semmer NK, Elfering A, Jacobshagen N, Meier L. Does stress at work make you gain weight? A twoyear longitudinal study. Scandinavian Journal of Work, Environment \& Health. 2011;37(1):45-53.

43. Takaki J, Minoura A, Irimajiri H, Hayama A, Hibino Y, Kanbara S, Sakano N, Ogino K. Interactive Effects of Job Stress and Body Mass Index on Over-eating. Journal of Occupational Health. 2010;52(1):66-73.

44. Kivimaki M, Nyberg ST, Batty GD, Fransson EI, Heikkila $\mathrm{K}$, Alfredsson L, et al. Job strain as a risk factor for coronary heart disease: a collaborative meta-analysis of individual participant data. The Lancet. 2012;380(9852):1491-1497.

45. Theorell T, Hammarström A, Gustafsson PE, Hanson LM, Janlert U, Westerlund H. Job strain and depressive symptoms in men and women: a prospective study of the working population in Sweden. Journal of Epidemiology \& Community Health. 2014;68(1):78-82.

46. Thouchette E, Henegar A, Godart NT, Pryor L, Falissard B, Tremblay RE, Côté SM. Subclinical eating disorders and their comorbidity with mood and anxiety disorders in adolescent girls. Psychiatry Research. 2011;185(12):185-192.

47. Ivanova IV, Tasca GA, Hammond N, Balfour L, Ritchier K, Koszycki D, Bissada H. Negative affect mediates the relationship between interpersonal problems and binge-eating disorder symptoms and psychopathology in a clinical sample: a test of the interpersonal model. European Eating Disorders Review. 2015;23(2):133-138.

48. Solmi F, Hatch SL, Hotopf M, Treasure J, Micali N. Prevalence and correlates of disordered eating in a general population sample: the South East London Community Health (SELCoH) study. Social Psychiatry and Psychiatric Epidemiology. 2014;49(8):1335-1346.

49. Grande AJ, Loch MR, Guarido EA, Costa JBY, Grande GC, Reichert FF. Comportamentos relacionados à saúde entre participantes e não participantes da ginástica laboral. Revista Brasileira de Cineantropometria \& Desempenho Humano. 2011;13(2):131-137. 
50. Alvarenga MS, Koritar P, Pinzon VD, Figueiredo M, Fleitlichbilyk, B, Philippi ST, Scagliusi FB. Validation of Disordered Eating Attitude Scale for adolescents. Jornal Brasileiro de Psiquiatria, Rio de Janeiro. 2016;65(1):3643.

51. O’Neil P, Currey H, Malcolm R, Sexauer J, Riddle F, Taylor C. Development and validation of the eating behavior inventory. Journal of Behavioral Assessment. 1979;1(2):123-132.

52. Unick JL, Jakicic JM, Marcus BH. Contribution of Behavior Intervention Components to 24-Month Weight Loss. Medicine \& Science in Sports \& Exercise. 2011;42(4):745-753.

53. Garner DM, Garfinkel PE. The Eating Attitudes Test: an index of the symptoms of anorexia nervosa. Psychological Medicine. 1979;9(2):273-279.

54. Garner DM, Olmsted MP, Bohr Y, Garfinkel PE. The Eatting Attitudes Test: psychometric features and clinical correlates. Psychological Medicine. 1982;12(4):871-878.

55. Stunkard AJ, Messick S. The three-factor questionnaire to measure dietary restraint, disinhibition and hunger. Journal of Psychosomatic Research. 1985;29(1):71-83.

56. Karlsson J, Persson LO, Sjoèstroèm L, Sullivan M. Psychometric properties and factor structure of the Three-Factor Eating Questionnaire (TFEQ) in obese men and women. Results from the Swedish Obese Subjects (SOS) study. International Journal of Obesity. 2000;24(12):1715-1725.

57. Natacci LC, Ferreira Júnior M. The three factor eating questionnaire - R21: tradução para o português e aplicação em mulheres brasileiras. Revista de Nutrição. 2011;24(3):383-394.

58. Van Strien T, Frijters J, Bergers G, Defares P. The Dutch Eating Behavior Questionnaire (DEBQ) for assessment of restrained, emotional, and external eating behavior. International Journal of Eating Disordes. 1986;5:295-315.

59. Wardle J. Eating style: A validation study of the Dutch Eating Behavior Questionnaire in normal subjects and women with eating disorders. Journal of Psychosomatic Research. 1987;31(2):161-169.

\section{Como citar este artigo:}

Souza MPG, Sampaio R, Cavalcante ACM, Arruda SPM, Pinto FJM. Comportamento alimentar e fatores associados em servidores: contribuições para a saúde coletiva. Rev. Aten. Saúde. 2020; 18(63): 99-109. 